$\mathcal{G}_{\text {https://doi.org/10.3765/sp.13.7 }}^{\text {Semantics \& Pragmatics Volume 13, Article 7: 1-27, } 2020}$

\title{
The semantics and pragmatics of nouns in concealed questions*
}

\author{
Hana Kalpak \\ Stockholm University
}

Submitted 2019-02-25 / First decision 2019-08-26 / Revision received 2020-01-03 / Second decision 2020-07-22 / Revision received 2020-07-24 / Accepted 2020-07-24 / Published 2020-07-29 / Final typeset 2022-04-28

\begin{abstract}
Concealed questions (CQs) are determiner phrases that are naturally paraphrased as embedded questions: I know your age reads as I know what your age is. The availability of CQ readings of determiner phrases has recently been taken to depend on whether the DP head noun is relational (i.e., two-place, like age or capital) or sortal (one-place, like brick or city) (Barker 2016). This generalization is based on the observation that many definite DPs lack salient concealed question-readings when their head noun is an unmodified sortal. Relatedly, Frana $(2013,2017)$ argues that, while quantified DPs have multiple concealed question-readings when the head noun is relational, they have only one when the head noun is sortal. This remark brings together noted counterexamples to both generalizations, and argues on their basis for a pragmatic account of the availability of concealed question readings of determiner phrases. The proposed account refines the analysis of concealed questions developed by Aloni \& Roelofsen (2011) through integrating it with (i) a standard analysis of the semantic distinction between relational and sortal nouns, and (ii) Barker's (2016) ideas about the role of salient sets of alternatives in licensing CQ interpretation. The resulting account is shown to deliver correct predictions for the counterexamples to both semantic generalizations, while simultaneously offering an explanation of the strong, yet imperfect, correlation of CQ readings of determiner phrases with a relational head noun.
\end{abstract}

Keywords: concealed questions, relational nouns, conceptual covers, alternatives

* I am very grateful to Maria Aloni for providing essential input at the formative stage of this work. I also want to thank Jonathan Pesetsky, Dean McHugh and Morwenna Hoeks for discussion and native speaker judgments, and the audience at CLS 54 for helpful commentary on early versions of the ideas presented here, which appeared in Kalpak 2019. The paper has benefitted greatly from comments and suggestions from three anonymous reviewers for Semantics \& Pragmatics, as well as from the editor, Kai von Fintel. All remaining errors are mine.

(C)2020 Hana Kalpak

This is an open-access article distributed under the terms of a Creative Commons Attribution License (https://creativecommons.org/licenses/by/3.o/). 
Hana Kalpak

\section{Introduction}

Concealed questions (CQs) are determiner phrases that read as embedded interrogatives (Baker 1968):

(1) I know the time.

$\approx$ I know what the time is.

Some determiner phrases are better concealers of questions than others. While the sentences in (2) are readily interpreted as containing the outlined concealed questions, the sentences in (3) are difficult to make sense of at all, let alone as containing concealed questions.

(2) a. Kim knows the capital of Italy.

(Heim 1979)

$\approx$ Kim knows what the capital of Italy is.

b. Max found out Sam's age.

(Barker 2016)

$\approx$ Max found out what Sam's age was.

c. They revealed the winner of the contest.

(Heim 1979) $\approx$ They revealed who the winner of the contest was.

(3) a. \#Kim knows the shoes.

(Frana 2006)

b. \#Max found out Sam's brick.

(Barker 2016)

c. \#They revealed the semanticist.

(Nathan 2006)

In these examples, the ability of a DP to read as a concealed question correlates with the semantic type of the DP's head noun. The sentences in (2) feature DPs with relational head nouns: capital expresses a relation between countries and cities, age a relation between individuals and numbers, and winner a relation between individuals and contests. ${ }^{1}$ The sentences in (3) instead feature DPs with sortal nouns, denoting sets of individuals. Data conforming to this contrast has recently been taken to indicate that the ability of a DP to read as a CQ is semantically determined. Building on Löbner 1981 and Nathan 2006, Barker (2016) argues that a DP requires a relational head noun, or particular types of noun modification, to be interpretable as a CQ. Frana $(2013,2017)$ defends a weaker, but related generalization, according to which only certain CQ readings of quantified DPs depend on the head noun type.

In this remark, I argue on the basis of examples like (4) for an analysis of CQs that makes more nuanced predictions about their distribution.

1 Time in (1) can likewise be relational, reading as the time of this utterance (Barker 2016: §9). 
Nouns in concealed questions

(4) I know your card. $\approx$ I know what (suit and rank) your card is. ${ }^{2}$

Card is an unmodified sortal noun, and the felicity of (4) therefore shows that it is not necessary for a DP to have a relational head noun, or feature particular modifiers, in order to have a CQ reading. Conversely, examples like (5) show that this condition is also not sufficient: mother is relational.

(5) \#Alex knows the mother.

(Frana 2006)

Analogous examples (Section 3) break the correlation between relational head nouns and CQ readings of quantified DPs proposed by Frana $(2013,2017)$.

Examples like these are not new to the literature, but underappreciated. Like Frana (2006) and Schwager (2008) I treat them as counterexamples to semantic explanations of CQ availability, and conclude that we instead need a pragmatic explanation, both for definite and quantified CQs. Still, a satisfactory account ought to explain the strong tendency of relational nouns to aid CQ interpretation. On the analysis to be proposed here, CQ availability is taken to depend foremost on the salience of a method of identification of individuals in a contextually determined domain of alternatives. This will predict that just about any DP can have a CQ reading in the appropriate context, but also that DPs headed by relational nouns have a larger set of such appropriate contexts: the domain of a relational noun always provides a suitable domain of alternatives.

Barker (2016) originally proposed that concealed question interpretation depends on the availability of a domain of alternatives, assumed to be encoded in the semantics of a DP's head noun. The present paper will argue that the alternative domain is instead pragmatically determined: while the DP head noun provides a default domain, this can be overridden in order to ensure a non-trivial CQ meaning.

The import of methods of identification for CQ interpretation is the key insight underlying both Schwager's (2008) and Aloni \& Roelofsen's (2011) formal semantics for concealed questions. However, both analyses ignore the semantic distinction between sortal and relational nouns. This prevents a satisfactory analysis of the full range of attested CQs, as well as an explanation of the link between relational nouns and CQ availability. The main contribution of this remark is to show that once we add a standard treatment of the relational-sortal distinction to Aloni \& Roelofsen's account, their analysis yields the correct predictions regarding the availability of CQ readings, and

2 Cf. Aloni \& Roelofsen's (2011) I know the winning card. 
their link to relational nouns. This also solves an independent problem for the original account, by avoiding recourse to derived conceptual covers for the interpretation of quantified CQs.

The paper is structured as follows. Section 2 outlines previous semantic explanations of CQ availability. Section 3 details the main counterarguments to such analyses, and motivates an alternative, pragmatic account. Section 4 makes minimal revisions to Aloni \& Roelofsen 2011 in order to account for the relevant data, and Section 5 outlines the predictions made by the revised account. Section 6 concludes.

\section{Background}

The idea that the type of a determiner phrase's head noun determines its suitability as a concealed question traces back at least to Löbner 1981. Löbner proposes that concealed questions express questions of the actual value of a "functional concept" (similar to an individual concept). For instance, (6) would express that Robin knows what the value of the functional concept expressed by the temperature of the water is at the world-time index of utterance:

(6) Robin knows the temperature of the water.

To Löbner, functional concepts are expressed by DPs built from functional head nouns, making such phrases interpretable as concealed questions.

A clarification of our assumed noun type taxonomy is in place. We follow the convention established in Partee 1997 and take sortal nouns to be nouns denoting sets of individuals, such as person, city, day and number. Relational nouns are nouns that denote relations over individuals, such as parent, capital, birthday and age. Intuitively, a parent is a person who stands in a particular relation to another person (their child). Likewise, a capital is a city that stands in a particular relation to a country, a birthday is a day that stands in a particular relation to an individual, and so forth. Given this semantic assumption, relational nouns are commonly treated as syntactically two-place, although their internal argument need not be overtly expressed (as in The capital is overpopulated.)

Among relational nouns, we further distinguish between functional and non-functional nouns (de Bruin \& Scha 1988). A functional noun is true of a unique external argument for each internal argument. This includes Löbner's temperature, but excludes, for instance, phone number (since one may 
Nouns in concealed questions

have multiple phone numbers). Functional nouns can be further divided into those expressing one-to-one functions, like nose (since no two individuals have the same nose) and those expressing functions that are merely onto, like temperature (since different objects may have the same temperature).

Many common examples of concealed questions contain DPs built from non-functional relational nouns:

(7) John knows Bill's phone number.

$\approx$ John knows what Bill's phone number is.

(Heim 1979)

Consequently, proponents of a semantic contrast typically depart from Löbner 1981 and assume that definite concealed questions include determiner phrases built from relational, rather than only functional, head nouns. Certain types of modification are known to robustly aid CQ interpretation of DPs headed by sortal nouns, notably modification by favorite, relative clauses, and superlatives.

(8) a. John's favorite drink is obvious.

(Heim 1979)

b. \#John's drink is obvious.

(9) a. Tell me the semanticist who teaches at UMass. (Nathan 2006)

b. \#Tell me the semanticist.

(10) a. John knows the largest town in Italy.

b. \#John knows the large town in Italy.

(Heim 1979)

Thus, the main contrast is taken to be between (i) DPs headed by sortal nouns lacking the above types of modification, and (ii) DPs headed either by relational nouns, or by suitably modified sortal nouns. The idea that this contrast is a result of semantic constraints on CQ formation has been motivated and formally implemented in particular by Nathan (2006) and Barker (2016).

Nathan derives concealed question-meanings from determiner phrases through two type-shifters: one that selects exclusively for relational nouns, and one that selects for sortal nouns featuring one of the above types of modification (Nathan 2006: §4). This treatment predicts that unmodified sortals cannot head concealed questions, and that all DPs formed from relational nouns can be concealed questions, since the type-shifters apply indiscriminately to objects of the relevant sort.

Nathan does not offer any independent reason not to also stipulate a typeshifter deriving CQ meanings from plain sortal nouns. Barker (2016) aims to provide such a reason. Barker argues for a treatment of interrogatives as hav- 
ing structured meanings; that is, as expressing foreground-background pairs in the sense of Krifka 2001. The foreground identifies a domain of alternatives, and the background specifies a property distinguishing among these alternatives. Intuitively, the question Which day is John's birthday? identifies the set of days (the foreground), and asks which member of this set has the property of being John's birthday (the background).

Under embedding, foreground-background denotations are converted into propositions through a type-shifter selecting for foreground-background pairs. Barker suggests that the same type-shifter is responsible for deriving question meanings from determiner phrases, to the effect that only DPs expressing foreground-background pairs are licit CQs. On Barker's semantics, relational nouns express foreground-background structures: a noun like birthday expresses a pair of the property of being a day (the foreground), and the property of standing in the born-on relation to some element of the foreground (the background).

(11) $\llbracket$ birthday $\rrbracket=\langle\llbracket$ day $\rrbracket, \llbracket$ born-on $\rrbracket\rangle=\{\langle x, \llbracket$ born-on $\rrbracket(x)\rangle \mid x \in \llbracket$ day $\rrbracket\}$

When heading a full DP, determiners, modifiers, and arguments are integrated compositionally into the foreground-background pair, with the result that the full DP expresses such a pair.

$$
\begin{aligned}
& \llbracket \text { John's birthday } \rrbracket=\langle\llbracket \text { day } \rrbracket, \llbracket \text { born-on } \rrbracket(\llbracket \text { John } \rrbracket)\rangle \\
& =\{\langle x, \llbracket \text { born-on } \rrbracket(\llbracket \text { John } \rrbracket)(x)\rangle \mid x \in \llbracket \text { day } \rrbracket\}
\end{aligned}
$$

In contrast, the meaning of a sortal noun, like day, is not lexically divisible into foreground-background properties. Assuming that determiners cannot create foreground-background structures, DPs headed by unmodified sortal nouns are predicted to be semantically unfit for interpretation as concealed questions. In contrast, DPs headed by relational nouns all denote foregroundbackground structures, and thereby fulfill the necessary condition for receiving a CQ interpretation.

Likewise, Barker argues that both relative clauses and the modifier $\mathrm{fa}$ vorite create foreground-background structures from their arguments, thus predicting that DPs formed from NPs containing these modifiers can be selected by the question type-shifter. 
Nouns in concealed questions

\subsection{Quantified concealed questions}

While both Nathan (2006) and Barker (2016) focus on definite and possessive DPs, their accounts have implications also for quantified concealed questions, like the one in (13).

(13) John knows every phone number.

(Heim 1979)

As Heim (1979) observed, quantified CQs tend to be ambiguous between a set reading and a pair-list reading. ${ }^{3}$ For instance, (13) may mean either that John knows a set of phone numbers, without knowing whose they are (the set reading), or that John knows, for every person in some domain, their phone number (the pair-list reading).

Not all quantified DPs allow these readings readily. Barker (2016) judges that quantified DPs with sortal head nouns, like that in (14), lack both CQ readings:

\section{(14) Ann guessed every number.}

This follows from Barker's account of concealed questions: quantified DPs denote foreground-background structures in order to be shiftable into questions, but only those with a relational head noun, or a "relationalizing" modifier like favorite, fulfill this requirement.

Barker's judgment is controversial. Frana $(2013,2017)$ agrees that quantified DPs headed by sortal nouns lack pair-list readings, but argues that they still have set readings:

(15) Kim knows every European country.

Set reading: For every country $x$, Kim knows whether $x$ is a European country.

Frana offers a comprehensive account of quantified CQs that predicts this generalization. While the details of her analysis must be left out here for reasons of space, it should be noted that her account predicts that set readings are readily available also for DPs headed by unmodified sortal nouns, like (14) (as noted in Barker 2016: §8.4).

3 This terminology is Roelofsen \& Aloni's (2008), not Heim's. 
Hana Kalpak

\section{Counterexamples to a semantic contrast}

Semantic analyses are guided by the observation that often, the interpretation of a DP as a concealed question correlates with the head noun being relational. Yet, the literature contains several examples of CQs which break this pattern. We noted in the introduction that some definite DPs formed from relational nouns lack immediate concealed question readings:

$$
\begin{aligned}
& \text { a. \#Ann found out the truck's carburetor. } \\
& \text { b. \#Alex guessed Kim's nose. } \\
& \text { c. \#They revealed Robin's mother. }
\end{aligned}
$$

(Frana 2006)

This observation carries over to the case of quantified concealed questions. The below examples seem to lack both straightforward set and pair-list readings:

(17) a. \#Ann found out every carburetor.

b. \#Alex guessed every nose.

c. \#They revealed every mother.

This shows that relationality of the head noun, or noun phrase, is not sufficient to license the relevant CQ readings neither of definite nor of quantified DPs. Both Nathan (2006) and Barker (2016) acknowledge this, but leave an explanation for future work. Frana $(2013,2017)$ does not address examples of the kind in (17), and therefore does not predict any restrictions on the ability of relational nouns to allow pair-list readings.

Given the apparent oddness of examples like (16), Barker suggests that the ability to express a foreground-background structure is merely a necessary condition for a DP to read as a CQ. But there are counterexamples to this claim, too. Frana (2006) observes that even a sentence like the intuitively odd (18) may receive a CQ reading through a sufficiently rich specification of utterance context.

(18) ??I only knew the shoes.

Frana's exemplifies with a quiz show, in which the contestants are challenged to visually memorize a number of items, with the goal of later being able to tell from pictures of parts of the same objects, which object each picture displays. If the set of objects includes a pair of shoes, a contestant may felic- 
Nouns in concealed questions

itously use (19) to mean that 'I only knew what the shoes were', in the sense of knowing only which picture portrayed part of the shoes.

(19) I only knew the shoes.

$\approx$ I only knew what the shoes were (e.g., the object portrayed in picture A).

Similar contexts allow both set and pair-list readings of quantified DPs. (20) is the caption of a talkshow clip in which Nate is shown the silhouettes of various U.S. states, and correctly gives the name of the state for each silhouette. ${ }^{4}$

(20) Nate is only five years old, but he knows every state and where the capitals are.

Set reading: For every (U.S.) state $x$, Nate knows whether $x$ is a state. Pair-list reading: For every (U.S.) state $x$, Nate knows what the silhouette of $x$ is.

Other examples do not even require explicit specification of context. (21) (a variation on Aloni \& Roelofsen's (2011) I know the winning card) and (22) are contextualized by informants to read as outlined, even when presented without backdrop:

(21) I know your card. $\approx$ I know what (suit/rank) your card is.

(22) I will guess every card. $\approx$ For every card $x$, I will guess the suit/rank of $x$.

Thus, even without modification, a sortal noun can head a concealed question. These observations together indicate that CQ availability cannot be determined by some semantic property distinguishing unmodified sortals from modified sortals and relational nouns: the contrast between "good" and "bad" concealers of questions cuts across this distinction.

\subsection{Towards a pragmatic account}

The observation that contextual specification may make CQ readings available invites a pragmatic, rather than semantic, account of the availability of these readings. To my knowledge, the only attempt at explaining the ability

4 At the time of writing, the clip with caption is available at https://www.facebook.com/ ellentv/videos/10155496362772240/. 
of Frana's (2006) context to ameliorate (23) comes from Magda Kaufmann in Schwager 2008.

(23) I only knew the shoes.

Kaufmann proposes that a DP can only read as a CQ provided a non-trivial, contextually salient method of identification of a relevant set of individuals, including the DP's referent. This builds on Aloni 2001, which proposed the same constraints for overt questions. Such a method is typically one like naming, ostension, or definite description, and is non-trivial insofar as it yields a non-trivial CQ meaning (excluding e.g., I only knew that the shoes were the shoes). Frana's context makes salient a particular set of individuals the set of objects to be memorized - and a visual method of identifying its members, as the object depicted in picture one, the object depicted in picture two, and so forth.

Kaufmann notes that the need for contextual suppletion of a method of identification distinguishes a construction like (23) from one like (24).

(24) Kim knows the capital of Italy.

There is a context-independent method of identifying the members of the set of capitals; identification by naming. This, Kaufmann suggests, is the reason that (24) has a CQ reading out of the blue, while (23) needs a context like Frana's for such a reading to appear.

Kaufmann's suggestion is promising, but by itself fails to explain why (24) is felicitous, while (25) is not: cities do have names.

(25) \#Sam guessed the city.

There is nevertheless a straightforward explanation of the infelicity of (25), which is perfectly compatible with Kaufmann's suggestion. By the uniqueness presupposition of the definite article, (25) presupposes that there is a unique (contextually salient) city. This presupposition is not satisfied in your average context of communication. Satisfying it moreover contradicts taking the set of cities to be the set of individuals requiring a method of identification. Thus, a construction like (25) will need appropriate contextualization in order to ensure that its uniqueness presupposition is satisfied, and to make salient a set of individuals - including the DP referent - that is not merely (a subset of) the head noun extension. This is precisely what Frana's quiz-show context does for (23). 
Nouns in concealed questions

It is never discussed in Schwager 2008 how the set of individuals requiring identification is selected. In contrast, Barker (2016) argued that a domain of alternatives is provided as the "foreground" of a relational noun (or modified sortal). The context-dependence of constructions like (23) and (25) show that Barker's assumption is somewhat too strong: to interpret these sentences as expressing defined but non-trivial CQs, we cannot treat the alternative domain as a set of shoes or cities - it needs to be provided by context.

The upcoming sections will spell out a formal treatment of CQs reflecting the following hypothesis: A definite DP has a CQ reading in a given context of communication $c$ just in case in $c$, the DP is defined, and there is a nontrivial method of identification of a salient domain of alternatives, including the referent of the DP. We thus follow Barker (2016) in assuming dependence on an alternative domain, but require only that this domain be salient, not that it is encoded in the semantics of the DP head noun. This will allow alternatives to be contextually provided when required to produce a consistent but non-trivial question denotation.

CQ readings of possessive DPs will likewise be taken to depend on the availability of methods of identification over domains of alternatives, but also on the salience of a suitable relation between possessor and possessee(s). The latter was not considered by Schwager (2008).

Finally, the requirement of methods of identification will allow us to explain the correlation between relational nouns and CQ interpretation of quantified DPs (also not discussed by Kaufmann), while predicting the exceptions. The idea that these exceptions require a pragmatic explanation has a precursor in Frana 2013: Footnote 2, where it is suggested that pair-list readings can be derived through coercing a relational interpretation of a non-relational NP. However, Frana does not discuss when and why such coercion is licensed. The account to be developed here will improve on her suggestion by proposing precise pragmatic licensing conditions for pair-list readings, thereby explaining also the absence of such readings for certain quantified DPs headed by relational nouns (e.g., (17)).

\section{The proposal}

We take as our starting point the formal analysis of concealed questions proposed in Aloni \& Roelofsen 2011. Their analysis follows Schwager's (2008) 
in modeling concealed questions as dependent on methods of identification, but unlike the latter extends also to quantified concealed questions. ${ }^{5}$

\subsection{Aloni \& Roelofsen 2011}

On Aloni \& Roelofsen's account, concealed questions denote questions, derived from DP denotations via a dedicated type-shifter. ${ }^{6}$ Crucially for our purposes, Aloni \& Roelofsen's semantics of concealed questions employs conceptual covers. Conceptual covers are formalizations of methods of identification, like identification by naming, ostension, or definite description (Aloni 2001): Formally, a cover over a domain of individuals is a set of individual concepts such that each individual is identified by exactly one concept in each world:

Definition 1 (Conceptual covers) Given a set of worlds $W$ and a domain of individuals $D$, a conceptual cover $C C$ based on $(W, D)$ is a set of individual concepts, such that:

$$
\forall w \in W: \forall d \in D: \exists ! c \in C C: c(w)=d
$$

To illustrate, consider a domain of two individuals; Kim and Sam. The set of the individual concepts expressed by the names Kim and Sam is a conceptual cover over this domain. In context, other methods of identification may be salient. If Kim and Sam are perceptually available, they may be identified ostensively, by individual concepts such as those expressed by the person to the right, the person to the left. The set of these concepts constitutes another, non-equivalent, conceptual cover over our two-person domain. In contrast, the set of the concepts expressed by Kim and the person to the left, would not constitute a conceptual cover over this domain: at worlds where Kim stands to the left, Sam is not picked out, and Kim is picked out twice.

To capture the sensitivity of the interpretation of concealed questions to such methods of identification, we integrate conceptual covers into a standard language of first order logic, enriched with a ' $\iota$ '- operator and a question operator '?'. A model $M$ in this language will be a quadruple $(W, D, I, C)$, where $W$ is a set of possible worlds, $D$ a set of individuals, $I$ a (world-dependent)

5 For general motivations for this pragmatic approach to CQs, see especially Schwager 2008: §3 and Aloni \& Roelofsen 2011: §2.

6 For arguments in favor of assigning these constructions question denotations, see Aloni \& Roelofsen 2011: §2. 
Nouns in concealed questions

interpretation function, and $C$ a set of conceptual covers based on $(W, D)$. As usual, sentences are evaluated relative a model, world, and assignment function. The twist is that variables now range over conceptual covers, and not directly over $D$. The range of a given variable is contextually determined: for a model $M$, we define a set of cover resolution functions $R$ that map every variable to some conceptual cover in $M$.

Definition 2 (Cover resolution functions) Given a model $M=(W, D, I, C)$ and $a$ set $V$ of variables, a cover resolution function $R$ for $M$ is a function that maps every $x \in V$ to elements $C C \in C$.

The selection of a cover resolution function in a given context is governed by general pragmatic constraints. As discussed in detail by Aloni (2001), the function must map to covers that are relevant, informative and consistent relative the common ground. Among such functions, the default choice is the one mapping variables to the contextually most salient covers, typically

i. an ostensive cover, if the domain is perceptually available

ii. a naming cover, otherwise.

We will see more examples of these different types of covers in Section 5 .

Assignment functions map variables to individual concepts, given a cover resolution function:

Definition 3 (Assignment functions) Given a model $M=(W, D, I, C)$, a set $V$ of variables, and a cover resolution function $R$, an assignment function $g_{R}$ is a function mapping every variable $x \in V$ to some individual concept $c \in R(x)$.

Thus, at a given world, variables still pick out individuals, though they do so via individual concepts:

\section{Definition 4 (Variable denotations)}

$$
\llbracket x \rrbracket_{M, w, g_{R}}=g_{R}(x)(w)
$$

Both quantifiers and the $\iota$-operator now range over conceptual covers, rather than over $D$ directly: 


\section{Definition 5 (Quantifiers)}

$$
\begin{gathered}
\llbracket \exists x . \varphi \rrbracket_{M, w, g_{R}}=1 \text { iff } \exists c \in R(x): \llbracket \varphi \rrbracket_{M, w, g_{R}[x / c]}=1 \\
\llbracket \forall x . \varphi \rrbracket_{M, w, g_{R}}=1 \text { iff } \forall c \in R(x): \llbracket \varphi \rrbracket_{M, w, g_{R}[x / c]}=1
\end{gathered}
$$

Definition 6 (The เ-operator) If there is a unique $c \in R(x)$ such that $\llbracket \varphi \rrbracket_{M, w, g_{R}[x / c]}=1$, then:

$$
\llbracket \iota x . \varphi \rrbracket_{M, w, g_{R}}=c(w)
$$

Otherwise, $\llbracket \iota x . \varphi \rrbracket_{M, w, g_{R}}$ is undefined.

Following Groenendijk \& Stokhof (1984), Aloni \& Roelofsen (2011) analyze questions as having the form ? $x . \varphi$, and as expressing, at a world $w$, the proposition corresponding to the true exhaustive answer to ? $x . \varphi$ in $w$. In the present framework, this proposition is determined relative a cover resolution function:

\section{Definition 7 (Questions)}

$$
\llbracket ? z . \varphi \rrbracket_{M, w, g_{R}}=\left\{v \mid \forall c \in R(z): \llbracket \varphi \rrbracket_{M, w, g_{R}[z / c]}=\llbracket \varphi \rrbracket_{M, v, g_{R}[z / c]}\right\}
$$

At a world $w$, ?z.p now denotes the set of worlds which agree with $w$ on which individual concepts pick out individuals for which $\varphi$ holds.

We illustrate the workings of this semantics by deriving the meaning of (26):

(26) Kim knows the capital of Italy. $m K_{k}($ (x.CAPITAL $(i)(x))$

Aloni \& Roelofsen's translation is indicated after $\rightsquigarrow . K_{k}$ is Kim's knowledge operator, with the semantics given in Definition 8 (for any agent $a$, where $E_{a}$ is the set of worlds epistemically accessible to $a$ ).

\section{Definition 8 (Knowledge)}

$$
\llbracket K_{a} \varphi \rrbracket_{M, w, g_{R}}=1 \text { iff } E_{a}(w) \subseteq \llbracket \varphi \rrbracket_{M, w, g_{R}}
$$

$K_{k}$ thus embeds proposition-denoting expressions, but the definite description the capital of Italy denotes an entity. To resolve the type mismatch, Aloni \& Roelofsen propose the type-shifter $\uparrow_{z, P}$ : 
Nouns in concealed questions

Definition 9 (The type-shifter $\uparrow_{z, P}$ )

$$
\uparrow_{z, P} \alpha={ }_{\text {def }} ? z . P(\alpha)
$$

$\alpha$ is here some entity-denoting expression, and $P$ is some contextually salient property, per default (i) the property of being identical to $z$, or (ii) the property expressed by $\alpha$ 's head NP. When $\alpha$ is definite or possessive, as in (26), the only default resolution of $P$ able to yield a non-trivial question denotation is identity: ${ }^{7}$

(27) $\quad K_{k}\left(\uparrow_{z, P}\right.$ lx.CAPITAL $\left.(i)(x)\right) \leadsto K_{k}(? z \cdot z=\iota x \cdot \operatorname{CAPITAL}(i)(x))$

On Aloni \& Roelofsen's analysis, $x$ and $z$ range over the naming cover over capitals:

$$
R(z)=R(x)=\{\text { Beijing, Washington DC, Berlin ... }\}
$$

Given this, (26) is evaluated as true at our world $w^{*}$ just in case at $w^{*}$, Kim knows that the actual world is such that the individual picked out by the name Rome is the capital of Italy:

$$
\begin{aligned}
& \llbracket K_{k}(? z . z=\iota x . \operatorname{CAPITAL}(i)(x)) \rrbracket_{M, w^{*}, g_{R}}=1 \text { iff } \\
& E_{k}\left(w^{*}\right) \subseteq\{v \mid \text { Rome is the capital of Italy at } v\}
\end{aligned}
$$

This is Aloni \& Roelofsen's basic analysis of definite CQs. The different readings of quantified CQs are derived through different default resolutions of $P$ :

(29) Kim knows every capital. $\rightsquigarrow \forall x\left(\operatorname{CAPITAL}(x) \rightarrow K_{k}\left(\uparrow_{z, P} x\right)\right)$

The set reading is obtained by resolving $P$ to the property expressed by the DP noun phrase:

(30) Kim knows every capital. $\leadsto \forall x\left(\operatorname{CAPITAL}(x) \rightarrow K_{k}(\right.$ ?z.CAPITAL $\left.(x))\right)$

$P \mapsto \operatorname{EX}(\lambda x \cdot \operatorname{CAPITAL}(x))$

$A(x)=\llbracket$ CAPITAL $\rrbracket, R(x)=\{$ Washington DC, Beijing, Berlin $\ldots\}$

For every capital $x$, Kim knows whether $x$ is a capital.

7 The alternative is (i), whose denotation is obviously trivial regardless of selected range of $z$ and $x$ :

(i) $\quad K_{k}($ ?z.CAPITAL $(i)(\iota x . \operatorname{CAPITAL}(i)(x)))$ 
The derivation of pair-list readings involve the resolution of $P$ to the property of being identical to $z$ :

$$
\forall x\left(\operatorname{CAPITAL}(x) \rightarrow K_{k}(? z . z=x)\right)
$$

The evaluation of this formula requires two conceptual covers over $A(x)$ $(=A(z))$, which Aloni \& Roelofsen take to be the set of capitals. They solve this by allowing cover resolution functions to map to derived conceptual covers, obtained by applying the intension of a functional noun to a basic cover. By applying the function expressed by capital to the naming cover over countries, we obtain the cover \{the capital of the U.S.A., the capital of China... . Then, resolving $R(z)$ to the naming cover over capitals, and $R(x)$ to the derived cover, yields the interpretation of (31) as expressing that Kim knows, for each capital $x$, which country $x$ is the capital of.

The derived covers-approach both under- and overgenerates. It fails to predict the right pair-list readings for quantified DPs headed by non-functional relational nouns, like the one in (32):

(32) Sam knows every phone number. ${ }^{8}$

Since covers can be derived using functions that are not one-to-one, the account predicts spurious readings for CQs which themselves contain CQs (for discussion, see Aloni \& Roelofsen 2011: Appendix A2). The modifications made to Aloni \& Roelofsen's account in the upcoming subsection will allow us to account for pair-list readings without relying on derived covers.

\subsection{Our additions}

Aloni \& Roelofsen formalize relational nouns as one- or two-place predicates depending on whether the internal argument is overtly expressed, but are silent on how these readings are derivationally related. Since their focus is on constructions involving relational nouns, they offer no account of how possessive DPs featuring unmodified sortal nouns are composed. They assume that the domain of alternatives over which covers are defined always equals the extension of the DP head noun (under a one-place reading). But as noted in the discussion of (23) in Section 3, this domain can also be supplied by context. Our additions to Aloni \& Roelofsen's setup will therefore aim to

8 Aloni \& Roelofsen (2011) discuss this example, but erroneously treat phone number as functional. 
Nouns in concealed questions

refine the treatment of the relational-sortal distinction, of possessives, and of the domains of alternatives.

We will follow the common analysis of relational nouns as lexically twoplace, with one-place readings obtained by existential closure of the internal argument (Barker 2011). A type-shifter for this purpose is defined below.

\section{Definition 10 (Existential closure)}

$$
\mathrm{Ex}={ }_{\text {def }} \lambda R_{\langle e,\langle e, t\rangle\rangle} \lambda x \cdot \exists v R(v)(x)
$$

Sortal nouns receive the usual one-place treatment.

Following Partee (1997), we will assume that the internal structure of possessive constructions vary with the type of the head noun of the possessed NP. While relational nouns feature an inherent relation linking possessor to possessee, sortal nouns do not. Formally, constructions like 'Sam's NP' or 'the NP of Sam('s)' have the logical form

- $\iota x \cdot \mathrm{NP}(s)(x)$ when NP is relational

- $\iota x \cdot \mathrm{NP}(x) \wedge \pi(s)(x)$ when NP is sortal

where $\pi$ is a free relation variable, whose content needs to be pragmatically supplied. Typically, $\pi$ is resolved to an agentive or a control relation (Vikner \& Jensen 2002).

For instance, Kim's painting can denote a painting that is created by or owned by Kim. While such relations tend to be default, pragmatics can favor others, as in the interpretation of John's team as describing the team that John is a fan of (Partee 1997).

Aloni \& Roelofsen assume two default resolutions of the property $P$ in their type-shifter $\uparrow_{z, P}$ : the property of standing in the identity relation to $z$, and the property expressed by the argument's head NP. Intuitively, however, the presence of an unsaturated relational noun $R$ in the NP makes a further property salient: the property of standing in the relation expressed by $R$ to $z$. We will therefore assume that this resolution is a further default option for DPs with such head nouns.

We follow Barker (1995) and take sortal nouns to denote sets of individuals $x$, and relational nouns to denote sets of pairs of individuals $\langle x, y\rangle$, defined by conditions on the domains of $x, y$, and their relation. For instance, the meaning of birthday will be identified with the set in (33): 
The internal argument domain corresponds precisely to what Barker (2016) identifies as the foreground of the structured meaning of birthday; the property of being a day. We will assume with Aloni \& Roelofsen that such argument domains are the default choices for the domain of alternatives required to interpret a given CQ. Unlike Barker, however, we will allow these defaults to occasionally be overridden.

More generally, we will assume that each variable $x$ in a given DP is associated with an alternative domain $A(x) \subseteq D$, and that $R(x)$ must be based on $(W, A(x))$. The default choices of $A(x)$ derive from the semantics of the predicate applied to $x$ in the DP: when $x$ occurs as the internal (external) argument of a noun $N, A(x)$ is the internal (external) argument domain in $\llbracket N \rrbracket$. This holds also for sortal $N$, in which case the internal argument domain is $\llbracket N \rrbracket$ itself. ${ }^{9}$ However, if the default choice of $A(x)$ does not allow a choice of $R(x)$ obeying the conditions on relevance, informativity, and consistency required by Aloni (2001), resolution of $A(x)$ to some distinct, contextually salient set obeying these constraints will be licensed. ${ }^{10}$

In sum, our addition of an analysis of the relational-sortal distinction to Aloni \& Roelofsen's semantics have prompted us to assume an additional default resolution of $P$ in the type-shifter, and allowed us to incorporate a pragmatic variant of Barker's (2016) assumption regarding the source of the domain of alternatives. Note that these additions make no crucial change to the predictions for the possessive CQ discussed in the previous section:

$$
K_{k}(? z . z=\iota x . \operatorname{CAPITAL}(i)(x))
$$

While Aloni \& Roelofsen's take $R(x)$ and $R(z)$ to cover the set of capitals, we instead assume that $A(x)=A(z)=\llbracket$ city , making the natural choice of $R$ such that $R(x)=R(z)=$ the naming cover over cities. This does not affect the meaning assigned to (34), but will be seen to improve the predictions for plain definite and quantified DPs.

9 This is a simplification chosen for the cases at hand, where each $x$ is predicated over exactly once in the DP. The general case requires choosing the domain to be the intersection of the argument domains of each predicate applied to $x$ in the DP.

10 While there are many possible ways of salience of, I will restrict the discussion to cases featuring uncontroversial sources, like recent mention and perceptual availability (discussed further in Aloni 2001). 
Nouns in concealed questions

\section{Predictions}

This section outlines the predictions made by the extended account for definite, possessive, and quantified CQs with different types of head noun. The focus will lie on the contrast between unmodified sortal and relational nouns, reserving comments on modified cases for the final subsection.

\subsection{Plain definite DPs}

Recall our hypothesis: A definite DP has a CQ reading (in a context of communication $c$ ) just in case (in $c$ ) the DP is defined, and there is a non-trivial method of identification of a salient domain of alternatives. This is just as predicted by the modified version of Aloni \& Roelofsen's account. A construction featuring a relational noun has a CQ reading whenever there is a method of identification of the elements in the noun's internal argument domain. Given the naming-cover over cities, we predict that (35) has a CQ reading whenever the context features only one capital, for instance as Max knows which of the cities is the capital.

(35) Max knows the capital.

The account likewise explains the restricted availability of CQ readings for DPs built from sortal nouns, like (36).

(36) Kim knows the shoes. $\rightsquigarrow K_{k}(? z \cdot z=\iota x \cdot \operatorname{SHOES}(x))$

To evaluate this construction for a given $c$, the framework requires that in $c, \iota x \cdot \operatorname{SHOES}(x)$ is defined, and there is a salient set $A(z)$ (such that $A(z)=$ $A(x)$ ) over which we have a non-trivial, salient cover $R(z)$. Note that $A(z)$ cannot simply be a non-singleton subset of $\llbracket$ shoes $\rrbracket$ : this would contradict the assumption that $\iota x \cdot \operatorname{SHOES}(x)$ is defined, preventing a consistent choice of $R(z)$. Taking $A(z)$ to be a singleton subset of $\llbracket$ shoes $\rrbracket$ in contrast yields a trivial question denotation. Thus, $A(z)$ needs to be supplied by context.

Frana's (2006) context satisfies these requirements. Let $O$ be the set of objects depicted by the pictures shown in the quiz show (an intuitively salient and relevant set), and let $R$ map $z$ to the set of concepts expressed by the object depicted in picture $A$, the object depicted in picture B..., and so forth. If the shoes are depicted in picture A at our world $w^{*}$, then (36) expresses that Kim knows that the actual world is such that the shoes are picked out by the description the object depicted in picture A: 


$$
\begin{aligned}
& \llbracket K_{k}(? z . z=\iota x \cdot \operatorname{SHOES}(x)) \rrbracket_{M, w^{*}, g_{R}}=1 \text { iff } \\
& E_{s}\left(w^{*}\right) \subseteq\{v \mid \text { The object depicted in picture } A \text { is the shoes at } v\}
\end{aligned}
$$

Likewise, a construction like (38) will require a non-default choice of $A(z)$ (and $A(x)$ ), in order for the context to satisfy the requirement that $\iota x$. CITY $(x)$ is defined.

(38) Sam knows the city. $\rightsquigarrow K_{s}(? z \cdot z=\iota x \cdot \operatorname{CITY}(x))$

In the right context, we could have a choice of $A(z)=A(x)=\{\llbracket$ Italy $\rrbracket$, $\llbracket$ Rome $\rrbracket$, which together with the resolution $R(z)=R(x)=\{$ Italy, Rome $\}$ yields the interpretation of (38) as expressing that Sam knows which of Italy and Rome is a city.

This analysis improves on Aloni \& Roelofsen's (2011) by allowing domains of alternatives to be supplied by context, as required for non-contradictory readings of constructions like (36) and (38), and from the internal argument domain of a relational noun, as required for consistent reading of constructions like (35). The resulting treatment thereby captures the observation that DPs headed by sortal nouns read as CQs in the right context. The analysis still predicts that such DPs are more difficult to interpret as CQs than DPs headed by relational nouns: unlike the latter, the former always require a contextually supplied domain of alternatives.

\subsection{Possessive DPs}

Our formal treatment reflects the following hypothesis: A possessive DP has a CQ reading just in case the DP is defined, there is a non-trivial method of identification of a domain of alternatives, and there is a salient relation between possessor and possessee. For the construction Kim knows the capital of Italy, this is satisfied by many natural contexts: the alternative domain can felicitously be identified with the set of cities, the members of which are identifiable by names and relatable to countries by the relation expressed by capital.

DPs headed by sortal nouns will, in contrast, require some contextualization in order to resolve the free relation postulated for such constructions. Consider first a case where this relation is one of the pragmatic default choices:

(39) Max knows Kim's card. 
Nouns in concealed questions

On the reading of card as standard 52-deck playing card, we have a default conceptual cover the alternative domain $\llbracket$ card $\rrbracket$ : the cover consisting of the individual concepts expressed by the 52 possible combinations of suit and rank. Playing cards typically stand in a control-type relation to people - their purpose is to be featured in games, in which they are held by, dealt to, or picked by the game's participants. On this interpretation, (39) is true whenever Max knows the true answer to the question of which suit and rank the card Kim is controlling has, and false otherwise:

(40) Max knows Kim's card. $\rightsquigarrow K_{m}(? z \cdot z=\iota x \cdot \operatorname{CARD}(x) \wedge \pi(k)(x))$

$$
\begin{aligned}
& \pi \mapsto \text { CONTROLLED-BY } \\
& A(z)=A(x)=\llbracket \text { card } \rrbracket \\
& R(z)=R(x)=
\end{aligned}
$$

\{the ace of hearts, the ace of spades, the ace of clubs...\}

In other cases, we have a good, context-independent method of identifying the elements in the default domain of alternatives, but we lack the possibility of resolving the free relation to an agentive or control relation. (41) is a case in point: we typically identify days through dates or names of weekdays, but individuals cannot stand in control- or agentive relations to days.

(41) Alex knows Charlie's day.

Our account correctly predicts that such constructions are infelicitous out of the blue, but that a contextualization providing a candidate for the missing relation licenses a CQ reading of (41), for instance as in (42):

(42) Alex knows Charlie's day. $\rightsquigarrow K_{a}(? z \cdot z=\iota x \cdot \operatorname{DAY}(x) \wedge \pi(c)(x))$

$\pi \mapsto$ DISHES-ARE-DONE-ON-BY

$A(z)=A(x)=\llbracket$ weekday $\rrbracket$

$R(z)=R(x)=\{$ Monday, Tuesday, Wednesday ... $\}$

Alex knows which day is Charlie's day to do the dishes.

Conversely, while DPs headed by relational nouns feature an inherent relation, they may still fail to have CQ readings in the absence of a method of identification of a domain of alternatives. (43) is such an example:

(43) Alex guessed the car's carburetor. 
In a context where Alex is guessing names of a given car's perceptually available parts, however, (43) has a natural reading as (approximately) Alex guessed which of the objects is the car's carburetor.

The combination of Aloni \& Roelofsen's (2011) analysis with the standard treatment of possessive DPs headed by sortals as featuring a free relation allows us to model and interpret possessive CQs headed by sortal nouns, which escaped both Aloni \& Roelofsen and Barker (2016). The analysis predicts that possessive DPs headed by relational nouns are more likely (but not guaranteed) to have immediate CQ readings, in virtue of featuring inherent, rather than context-dependent, relations.

\subsection{Quantified DPs}

We follow Aloni \& Roelofsen's (2011) formalization of (44), except in our representation of capital as $\exists v \cdot \operatorname{CAPITAL}(v)(x)$.

(44) Kim knows every capital. $\leadsto \forall x\left(\exists v \cdot \operatorname{CAPITAL}(v)(x) \rightarrow K_{k}\left(\uparrow_{z, P} x\right)\right)$

Our additions do not affect the predictions for set readings, which are obtained by resolving $P$ to the property expressed by the DP noun phrase:

$$
\begin{aligned}
& \forall x\left(\exists v \cdot \operatorname{CAPITAL}(v)(x) \rightarrow K_{k}(? z . \exists v \cdot \operatorname{CAPITAL}(v)(x))\right) \\
& P \mapsto \operatorname{Ex}(\lambda x \lambda y . \operatorname{CAPITAL}(y)(x)) \\
& A(x)=\llbracket \operatorname{CITY} \rrbracket, R(x)=\{\text { New York, Shanghai, Hamburg } \ldots\} \\
& \text { For every capital } x, \text { Kim knows whether } x \text { is a capital. }
\end{aligned}
$$

The availability of this reading is not predicted to depend on the relationality of the head noun, but on the availability of a conceptual cover for $x$ over the default $A(x)$. Similarly to Aloni \& Roelofsen's original account, this delivers a set reading also for (46) (since countries are identifiable by name), but demands contextualization for a case like (47) (alternatively, a reading of apple as apple cultivar).

(46) Alex knows every country.

(47) ??Alex knows every apple.

Our analysis differs from Aloni \& Roelofsen's when it comes to pair-list readings, which no longer depend on so-called derived covers. Recall that, for a construction like (44), we predict that $P$ may by default resolve to the property of being a capital of $z$ : 
Nouns in concealed questions

$$
\begin{aligned}
& \forall x\left(\exists v \cdot \operatorname{CAPITAL}(v)(x) \rightarrow K_{k}(? z \cdot \operatorname{CAPITAL}(z)(x))\right) \\
& P \mapsto \lambda y \cdot \operatorname{CAPITAL}(z)(y)
\end{aligned}
$$

With the following default resolutions of the domains and their covers, we obtain the reading of (48) as For every capital $x$, Kim knows which country $x$ is the capital of, as desired.

- $A(x)=\llbracket \mathrm{CITY} \rrbracket, R(x)=\{$ New York, Shanghai, Hamburg ... $\}$

- $A(z)=\llbracket$ COUnTry $\rrbracket, R(z)=\{$ the U.S.A., China, Germany... $\}$

Unlike Aloni \& Roelofsen's original account, this also delivers pair-list readings for DPs headed by non-functional nouns, like (49): this is treated anal-

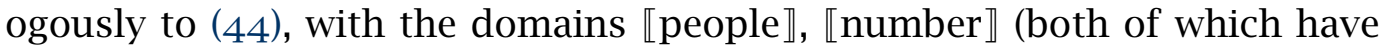
naming-covers).

(49) Sam knows every phone number.

Given the condition on non-triviality, the alternative resolution of $P$ to identity is only felicitous if we have two basic covers over the domain of the external argument of the noun. This is the only way in which a DP headed by an unmodified sortal can receive a pair-list reading. For instance, a construction like (50) has a straightforward pair-list reading in a context where a set of cards are identifiable in multiple ways, as is typically the case in a card-playing setting:

(50) Kim knows every card. $\rightsquigarrow \forall x\left(\operatorname{CARD}(x) \rightarrow K_{k}(? z . z=x)\right)$

$P \mapsto \lambda y \cdot z=y$

$A(z)=A(x)=\llbracket$ card $\rrbracket$

$R(x)=\{$ the ace of hearts, the ace of spades, the ace of clubs ... $\}$

$R(z)=\{$ the leftmost card, the rightmost card... $\}$

For every card $x$ on the table, Kim knows what the suit/rank of $x$ is.

Conversely, a quantified DP built from a relational noun may lack both an immediate set and pair-list reading:

(51) They revealed every mother.

We predict that provided a good context, (51) can receive a set reading (e.g., as For every mother $x$ in the room, they revealed whether $x$ is a mother) and a pair-list reading (e.g., as ...they revealed who $x$ is the mother of). 
Our analysis improves on Aloni \& Roelofsen's (2011) by predicting that only sortal nouns require multiple methods of identifying the same domain in order to produce pair-list readings. This demands a richer context, making quantified DPs headed by unmodified sortals more difficult to read this way. It also removes the need for derived covers, thereby capturing that pair-list readings appear also with non-functional relational nouns. These predictions largely align with those of Frana $(2013,2017)$, but derive from a more detailed hypothesis about the pragmatics of CQ readings, which unlike Frana's analysis offers an explanation also of why some sortal nouns lack immediate set readings, and some relational nouns lack immediate pair-list readings. ${ }^{11}$

\subsection{A note on modification}

The proposed account also squares well with the observation that CQ readings are facilitated by modification by favorite, relative clauses, and superlatives:

(52) a. John's favorite drink is obvious.

(Heim 1979)

b. \#John's drink is obvious.

(53) a. Tell me the semanticist who teaches at UMass. (Nathan 2006) b. \#Tell me the semanticist.

(54) a. John knows the largest town in Italy.

(Heim 1979)

b. \#John knows the large town in Italy.

Favorite supplies the relation needed to interpret the possessive DP in (52a), while the relative clause in (53a) allows the (otherwise inconsistent) resolution of the domain of alternatives to the extension of the noun semanticist. Finally, the superlative in (54a) radically increases the set of contexts in which the uniqueness presupposition of the DP is satisfied.

Unlike both Nathan (2006) and Barker (2016), however, we do not predict that these types of modification always license a CQ reading: constructions like Ann guessed the apple which is red/the reddest apple are clearly odd. A highly particular context is required in order to ensure a unique red(dest) apple, and provide a conceptual cover over a domain of apples (which in

11 The proposed analysis also shares Frana's assumption that noun transitivity facilitates pairlist readings, and her use of existential closure to derive set readings from transitive nouns. For reasons of space, I have left an overall comparison between the two accounts for future work. 
Nouns in concealed questions

contrast to semanticists lack a context-independent cover). This likewise suggests an explanation of the contrast between cases like ?Ann guessed the red apple and Ann guessed the right number, which puzzles Barker (2016: §9.2): a prenominal adjective may license CQ interpretation when the head noun has a context-independent method of identification, but not otherwise.

\section{Conclusion}

We have seen that, contrary to the predictions of Nathan 2006 and Barker 2016, the availability of CQ readings of determiner phrases does not depend solely on the type (relational versus sortal) of the head noun, or its modification. Even for DPs headed by unmodified sortal nouns, CQ readings can systematically be made available by contextual specification of a possessive relation (if needed), an alternative domain, and one or more methods of identification of the members of this domain. By incorporating a standard analysis of the relational-sortal distinction along with insights from Barker 2016 regarding domains of alternatives and their potential semantic sources, we got an analysis of CQs that predicts that relational head nouns aid CQ interpretation for definite, possessive, and quantified DPs alike, while offering an explanation of when and why this tendency fails.

\section{References}

Aloni, Maria. 2001. Quantification under conceptual covers. Amsterdam: University of Amsterdam dissertation.

Aloni, Maria \& Floris Roelofsen. 2011. Interpreting concealed questions. Linguistics and Philosophy 34(5). 443-478. https://doi.org/10.1007/s10988o11-9102-9.

Baker, Carl Leroy. 1968. Indirect questions in English. Urbana, IL: University of Illinois dissertation.

Barker, Chris. 1995. Possessive descriptions. Stanford, CA: Center for the Study of Language \& Information.

Barker, Chris. 2011. Possessives and relational nouns. In Claudia Maienborn, Klaus von Heusinger \& Paul Portner (eds.), Semantics: An international handbook of natural language meaning, vol. 2, 1109-1130. Berlin: de Gruyter. https://doi.org/10.1515/9783110255072.1109.

Barker, Chris. 2016. Why relational nominals make good concealed questions. Lingua 182. 12-29. https://doi.org/10.1016/j.lingua.2016.01.002. 
de Bruin, Jos \& Remko Scha. 1988. The interpretation of relational nouns. Association for Computational Linguistics (ACL) 26. 25-32. https://doi. org/10.3115/982023.982027.

Frana, Ilaria. 2006. The de re analysis of concealed questions: A unified approach to definite and indefinite concealed questions. Semantics and Linguistic Theory (SALT) 16. 17-34. https://doi.org/10.3765/salt.v16io.2951.

Frana, Ilaria. 2013. Quantified concealed questions. Natural Language Semantics 21(2). 179-218. https://doi.org/10.1007/s11050-012-9089-y.

Frana, Ilaria. 2017. Concealed questions. Corby: Oxford University Press. https: //doi.org/10.1093/acprof:0so/9780199670925.001.0001.

Groenendijk, Jeroen \& Martin Stokhof. 1984. On the semantics of questions and the pragmatics of answers. Amsterdam: University of Amsterdam dissertation.

Heim, Irene. 1979. Concealed questions. In Rainer Bäuerle, Urs Egli \& Arnim von Stechow (eds.), Semantics from different points of view, 51-6o. Berlin, Heidelberg: Springer. https://doi.org/10.1007/978-3-642-67458-7-5.

Kalpak, Hana. 2019. Sortal versus relational nouns in concealed questions. Chicago Linguistic Society (CLS) 54. 325-340.

Krifka, Manfred. 2001. For a structured meaning account of questions and answers. In Caroline Féry \& Wolfgang Sternefeld (eds.), Audiatur vox sapientia: Festschrift for Arnim von Stechow, 287-319. Berlin: Akademie Verlag. https://doi.org/10.1515/9783050080116.287.

Löbner, Sebastian. 1981. Intensional verbs and functional concepts: More on the "rising temperature" problem. Linguistic Inquiry 12(3). 471-477. http: //www.jstor.org/stable/4178235.

Nathan, Lance Edward. 2006. On the interpretation of concealed questions. Cambridge, MA: Massachusetts Institute of Technology dissertation. http: //hdl.handle.net/1721.1/37423.

Partee, Barbara. 1997. Uniformity vs. versatility: The genitive, a case study. In Johan van Benthem \& Alice van ter Meulen (eds.), The handbook of logic and language, 464-470. Amsterdam: Elsevier. https://doi.org/10.1016/ B978-044481714-3/50011-4.

Roelofsen, Floris \& Maria Aloni. 2008. Perspectives on concealed questions. Semantics and Linguistic Theory (SALT) 18. 619-636. https://doi.org/10. 3765/salt.v18io.2500.

Schwager, Magdalena. 2008. Keeping prices low: An answer to a concealed question. Sinn und Bedeutung 12. 582-596. https://doi.org/10.18148/ sub/2008.v12io.708. 
Nouns in concealed questions

Vikner, Carl \& Per Anker Jensen. 2002. A semantic analysis of the English genitive: Interaction of lexical and formal semantics. Studia Linguistica 56(2). 191-226. https://doi.org/10.1111/1467-9582.00092.

Hana Kalpak

Department of Philosophy

Stockholm University

Universitetsvägen $10 \mathrm{D}$

10691 Stockholm, Sweden

hana.kalpak@philosophy.su.se 